gr-qc/0301084

\title{
Multidimensional Global Monopole and Nonsingular Cosmology
}

\author{
Kirill A. Bronnikov \\ Centre for Gravitation and Fundam. Metrology, VNIIMS, 3-1 M. Ulyanovoy St., Moscow 117313, Russia; \\ Institute of Gravitation and Cosmology, PFUR, 6 Miklukho-Maklaya St., Moscow 117198, Russia
}

\section{Boris E. Meierovich}

Kapitza Institute for Physical Problems, 2 Kosygina St., Moscow 117334, Russia

Email: meierovich@yahoo.com; http://geocities.com/meierovich/

\begin{abstract}
We consider a spherically symmetric global monopole in general relativity in $(D=d+2)$-dimensional spacetime. The monopole is shown to be asymptotically flat up to a solid angle defect in case $\gamma<d-1$, where $\gamma$ is a parameter characterizing the gravitational field strength. In the range $d-1<\gamma<2 d(d+1) /(d+2)$ the monopole space-time contains a cosmological horizon. Outside the horizon the metric corresponds to a cosmological model of KantowskiSachs type, where spatial sections have the topology $\mathbb{R} \times \mathbb{S}^{d}$. In the important case when the horizon is far from the monopole core, the temporal evolution of the Kantowski-Sachs metric is described analytically. The Kantowski-Sachs space-time contains a subspace with a $(d+1)$-dimensional Friedmann-Robertson-Walker metric, and its possible cosmological application is discussed. Some numerical estimations in case $d=3$ are made showing that this class of nonsingular cosmologies can be viable. Other results, generalizing those known in the 4-dimensional space-time, are derived, in particular, the existence of a large class of singular solutions with multiple zeros of the Higgs field magnitude.
\end{abstract}

\section{Introduction}

In our recent paper with E. Podolyak [1] we considered the general properties of global monopole solutions in general relativity, developing some earlier results (see 2, 3] and references therein). It was confirmed, in particular, that the properties of these objects are governed by a single parameter $\gamma$, squared energy of spontaneous symmetry breaking in Planck units. For $0<\gamma<1$, solutions with entirely positive (or entirely negative) Higgs field are globally regular and asymptotically flat up to a solid angle deficit. In the range $1<\gamma<3$, the space-time of the solutions remains globally regular but contains a cosmological horizon at a finite distance from the center. Outside the horizon the geometry corresponds to homogeneous anisotropic cosmological models of Kantowski-Sachs type, whose spatial sections have the topology $\mathbb{R} \times \mathbb{S}^{2}$. The nonzero symmetry-breaking potential can be interpreted as a time-dependent cosmological constant, a kind of hidden vacuum matter. The potential tends to zero at late times, and the "hidden vacuum matter" disappears. This solution with a nonsingular static core and a cosmological metric outside the horizon drastically differs from the standard Big Bang models and conforms to the ideas advocated by Gliner and Dymnikova [4] that the standard Big Bang cosmology could be replaced by a globally regular model. A possibility of a nonsingular isotropic cosmological model had been discussed by Starobinsky [5].

The lack of isotropization at late times did not allow us to directly apply the toy model of a global monopole to the early phase of our Universe. Though, this circumstance does not seem to be a fatal shortcoming of the model since the anisotropy of the very early Universe could be damped later by particle creation, and the further stages with low energy densities might conform to the standard isotropic Friedmann cosmology. Another idea is to add a comparatively small positive quantity $\Lambda$ to the symmetry-breaking potential (to "slightly raise the Mexican hat"). It can change nothing but the late-time asymptotic which will be de Sitter, corresponding to the added cosmological constant $\Lambda$. These ideas deserve a further study.

In this paper we study the gravitational properties of global monopoles in multidimensional general relativity. Such considerations can be of interest in view of numerous attempts to construct a unified theory using the ideas of supersymmetry in high dimensions. Objects like multidimensional monopoles, strings and other topological defects might form due to phase transitions in the early Universe at possible stages when the present three spatial dimensions were not yet separated from others, and a greater number of dimensions were equally important.

More specifically, we consider a self-gravitating hedgehog-type configuration of a multiplet of scalar fields with the Mexican-hat potential $V=(\lambda / 4)\left(\phi^{2}-\eta^{2}\right)^{2}$ in a $D$-dimensional space-time with the structure $\mathbb{R}_{t} \times \mathbb{R}_{\rho} \times \mathbb{S}^{d}$ $(d=D-2)$, where $\mathbb{R}_{\rho}$ is the range of the radial coordinate $\rho$ and $\mathbb{R}_{t}$ is the time axis. The properties of such objects generalize in a natural way the results obtained in Ref. [1] and earlier papers (e.g., [2, 3]). Thus, for small values of 
the parameter $\gamma=8 \pi G \eta^{2}$ characterizing the gravitational field strength, the solutions are asymptotically flat up to a solid angle deficit. Within a certain range $d-1<\gamma<\bar{\gamma}(d)$ the solutions are nonsingular but contain a Killing horizon and a cosmological metric of Kantowski-Sachs type outside it. In the important case when the horizon is far from the monopole core, the temporal evolution of the Kantowski-Sachs metric is described analytically. The upper bound $\bar{\gamma}(d)$, beyond which there are no static solutions with a regular center, is also found analytically.

The above description concerned solutions with totally positive (or totally negative) scalar field magnitude $\phi$. As in [1, we here also find a class of solutions with any number $n$ of zeros of $\phi(r)$, existing for $\gamma<\gamma_{n}(d)$, where the upper bounds $\gamma_{n}$ are found analytically. All solutions with $n>0$ describe space-times with a regular center, a horizon and a singularity beyond this horizon.

We also discuss a possible cosmological application of multidimensional global monopoles, which can be of particular interest in the case of 5-dimensional space-time with 3-dimensional spheres $\mathbb{S}^{d}$. In this case the Kantowski-Sachs type model outside the horizon has the spatial topology $\mathbb{R} \times \mathbb{S}^{3}$. It is anisotropic in 4 dimensions but the 3-dimensional spheres $\mathbb{S}^{3}$ are isotropic. The anisotropy is thus related only to the fourth coordinate $t$, which is spatial outside the horizon and is a cyclic variable from the dynamical viewpoint. If we identify $\mathbb{S}^{3}$ with the observed space, ignoring the extra coordinate, we arrive at a closed cosmological model, with the Friedman-Robertson-Walker line element in the ordinary $(3+1)$-dimensional space-time.

A natural question arises: why is the fourth spatial dimension unobservable today? An answer cannot be found within our macroscopic theory without specifying the physical nature of vacuum. The conventional Kaluza-Klein compactification of the extra dimension on a small circle is not satisfactory in our case since it leads to a singularity at the horizon (as will be demonstarted in Sec. 3). So we leave this question open and note that the global monopole model has a chance to describe only the earliest phase of the cosmological evolution. Its later stages should involve creation of matter and a sequence of phase transitions possibly resulting in localization of particles across the $t$ direction. We then obtain a model with a large but unobservable extra dimension, similar in spirit to the widely discussed brane world models, see the reviews 6, 7, 8 and references therein.

The solutions of interest appear when the symmetry breaking scale $\eta$ is large enough, and one may suspect that quantum gravity effects are already important at this energy scale. We show in Sec. 2.3 that this is not the case when the monopole core radius is much greater than the Planck length: the curvature and energy scales are then in the whole space much smaller than their Planckian values.

The existence of nonsingular models of the early Universe on the basis of classical gravity supports the opinion that our Universe had never undergone a stage described by full quantum gravity. Apart from those discussed here, such models are now rather numerous (1, 4, 9, 10, see also refrences therein). All of them are evidently free of the long-standing problems of the standard Big Bang cosmology connected with the existence of multiple causally disconnected regions [11, 12].

The paper is organized as follows. In Sec. 2 we analyze the properties of a global monopole in $D=d+2$ dimensions (one time coordinate and $d+1$ spatial coordinates). It is a generalization of our previous results [1]. The particular case $d=3$ is studied in more detail in Sec. 3 along with its possible cosmological application. Unless otherwise indicated, we are using the natural units $\hbar=c=1$.

\section{Multidimensional global monopole}

\subsection{General characteristics}

The most general form of a static, spherically symmetric metric in $D=d+2$ dimensions is

$$
d s^{2}=\mathrm{e}^{2 F_{0}} d t^{2}-\mathrm{e}^{2 F_{1}} d \rho^{2}-\mathrm{e}^{2 F_{\Omega}} d \Omega^{2} .
$$

Here $d \Omega^{2}=d \Omega_{d}^{2}$ is a linear element on a $d$-dimensional unit sphere, parametrized by the angles $\varphi_{1}, \ldots, \varphi_{d}$ :

$$
d \Omega_{d}^{2}=d \varphi_{d}^{2}+\sin ^{2} \varphi_{d}\left(d \varphi_{d-1}^{2}+\sin ^{2} \varphi_{d-1}\left(d \varphi_{d-2}^{2}+\ldots+\sin ^{2} \varphi_{3}\left(d \varphi_{2}^{2}+\sin ^{2} \varphi_{2} d \varphi_{1}^{2}\right) \ldots\right)\right) ;
$$

$F_{0}, F_{1}$ and $F_{\Omega}$ are functions of the radial coordinate $\rho$ which is not yet specified. The nonzero components of the Ricci tensor are (the prime denotes $d / d \rho$ )

$$
\begin{aligned}
& R_{0}^{0}=\mathrm{e}^{-2 F_{1}}\left[F_{0}^{\prime \prime}+F_{0}^{\prime}\left(F_{0}^{\prime}+d F_{\Omega}^{\prime}-F_{1}^{\prime}\right)\right] \\
& R_{\rho}^{\rho}=\mathrm{e}^{-2 F_{1}}\left[d F_{\Omega}^{\prime \prime}+F_{0}^{\prime \prime}+d F_{\Omega}^{\prime 2}+F_{0}^{\prime 2}-F_{1}^{\prime}\left(F_{0}^{\prime}+d F_{\Omega}^{\prime}\right)\right] \\
& R_{2}^{2}=\ldots=R_{d+1}^{d+1}=-(d-1) \mathrm{e}^{-2 F_{\Omega}}+\mathrm{e}^{-2 F_{1}}\left[F_{\Omega}^{\prime \prime}+F_{\Omega}^{\prime}\left(F_{0}^{\prime}+d F_{\Omega}^{\prime}-F_{1}^{\prime}\right)\right]
\end{aligned}
$$

A global monopole with a nonzero topological charge can be constructed with a multiplet of real scalar fields $\phi^{a}$ 
$(a=1,2, \ldots, d+1)$ comprising a hedgehog configuration in $d+1$ spacial dimensions ${ }^{1}$ :

$$
\phi^{a}=\phi(\rho) n^{a}\left(\varphi_{1}, \ldots, \varphi_{d}\right),
$$

where $n^{a}\left(\varphi_{1}, \ldots, \varphi_{d}\right)$ is a unit vector $\left(n^{a} n^{a}=1\right)$ in the $(d+1)$-dimensional Euclidean target space, with the components

$$
\begin{aligned}
n^{d+1}= & \cos \varphi_{d}, \\
n^{d}= & \sin \varphi_{d} \cos \varphi_{d-1}, \\
n^{d-1}= & \sin \varphi_{d} \sin \varphi_{d-1} \cos \varphi_{d-2}, \\
& \cdots \\
n^{d-k}= & \sin \varphi_{d} \sin \varphi_{d-1} \ldots \sin \varphi_{d-k} \cos \varphi_{d-k-1}, \\
& \cdots \\
n^{2}= & \sin \varphi_{d} \ldots \sin \varphi_{2} \cos \varphi_{1}, \\
n^{1}= & \sin \varphi_{d} \ldots \sin \varphi_{2} \sin \varphi_{1} .
\end{aligned}
$$

The Lagrangian of a multidimensional global monopole in general relativity has the form

$$
L=\frac{1}{2} \partial_{\mu} \phi^{a} \partial^{\mu} \phi^{a}-V(\phi)+\frac{R}{16 \pi G},
$$

where $R$ is the scalar curvature, $G$ is the $D$-dimensional gravitational constant, and $V(\phi)$ is a symmetry-breaking potential depending on $\phi= \pm \sqrt{\phi^{a} \phi^{a}}$, and it is natural to choose $V$ as the Mexican-hat potential

$$
V=\frac{\lambda}{4}\left(\phi^{2}-\eta^{2}\right)^{2}=\frac{\lambda \eta^{4}}{4}\left(f^{2}-1\right)^{2} .
$$

We have introduced the normalized field magnitude $f=\phi(\rho) / \eta$ playing the role of the order parameter. The model has a global $S O(d+1)$ symmetry, which can be spontaneously broken to $S O(d) ; \eta^{2 / d}$ is the energy of symmetry breaking. We are using natural units, so that $\hbar=c=1$.

The Einstein equations can be written as

$$
R_{\mu}^{\nu}=-8 \pi G \widetilde{T}_{\mu}^{\nu}=-8 \pi G\left(T_{\mu}^{\nu}-\frac{1}{d} T \delta_{\mu}^{\nu}\right),
$$

where $T_{\mu}^{\nu}$ is the energy-momentum tensor. The nonzero components of $\widetilde{T}_{\mu}^{\nu}$ are

$$
\begin{aligned}
& \widetilde{T}_{0}^{0}=-\frac{2}{d} V, \\
& \widetilde{T}_{\rho}^{\rho}=-\mathrm{e}^{-2 F_{1}} f^{\prime 2}-\frac{2}{d} V, \\
& \widetilde{T}_{2}^{2}=\ldots=\widetilde{T}_{d+1}^{d+1}=-\mathrm{e}^{-2 F_{\Omega}} f^{2}-\frac{2}{d} V .
\end{aligned}
$$

Let us use the quasiglobal coordinate $\rho$ specified by the condition

$$
F_{0}+F_{1}=0
$$

which is a convenient gauge for spherically symmetric systems with Killing horizons. Introducing the functions $A(\rho)=\mathrm{e}^{2 F_{0}}=\mathrm{e}^{-2 F_{1}}$ and $r(\rho)=\mathrm{e}^{F_{\Omega}}$, we reduce the metric to the form

$$
d s^{2}=A(\rho) d t^{2}-\frac{d \rho^{2}}{A(\rho)}-r^{2}(\rho) d \Omega^{2},
$$

\footnotetext{
${ }^{1} \mathrm{~A} 7 \mathrm{D}$ universe with a global monopole with a hedgehog configuration of scalar fields only in three extra dimensions was recently considered by Benson and Cho 13. Our approach is different. We consider a hedgehog configuration in all $D-1$ space dimensions of the $D$-dimensional spacetime.
} 
and get the following equations:

$$
\begin{aligned}
\left(A r^{d} \phi^{\prime}\right)^{\prime}-d r^{d-2} \phi & =r^{d} \frac{\partial V}{\partial \phi}, \\
r^{\prime \prime} & =-\frac{8 \pi G}{d} r \phi^{\prime 2}, \\
\left(r^{d} A^{\prime}\right)^{\prime} & =-\frac{32 \pi G}{d} r^{d} V, \\
A\left(r^{2}\right)^{\prime \prime}-r^{2} A^{\prime \prime}-(d-2) r^{3} r^{\prime}\left(\frac{A}{r^{2}}\right)^{\prime} & =2\left(d-1-8 \pi G \phi^{2}\right)
\end{aligned}
$$

for the unknown functions $\phi(\rho), A(\rho)$ and $r(\rho)$. Only three of these four equations are independent: the scalar field equation (6) follows from the Einstein equations (7)-(9) due to the Bianchi identities.

Eqs. (6) - (8) have the same structure as Eqs. (13)-(15) in [1]. General properties of Eqs. (6) - (8) with arbitrary value of $d$ are the same as for $d=2$, and the classification of their solutions is also the same. In particular: if $V(\phi)>0$, the system with a regular center can have either no horizon or one simple horizon; in the latter case, its global structure is the same as that of de Sitter spacetime. Below we will concentrate our attention on the solutions belonging to class (a1) according to [1], i.e., $r(\rho)$ is monotonically growing from zero to infinity as $\rho \rightarrow \infty$, while $A(\rho)$ changes from $A=1$ at the regular center to $A_{\infty}<0$ as $\rho \rightarrow \infty$, with a cosmological horizon (where $A=0$ ) at some $\rho=\rho_{h}$.

Eq. (9) is a second-order linear inhomogeneous differential equation with respect to $A$. The corresponding homogeneous equation has the evident special solution $A(\rho)=$ const $\times r^{2}(\rho)$. This allows one to express $A(\rho)$ in terms of $r(\rho)$ and $\phi(\rho)$ in an integral form:

$$
A=C_{1} r^{2}-C_{2} r^{2} \int_{\rho}^{\infty} \frac{d \rho_{1}}{r^{d+2}\left(\rho_{1}\right)}+2 r^{2} \int_{\rho}^{\infty} \frac{d \rho_{1}}{r^{d+2}\left(\rho_{1}\right)} \int_{0}^{\rho_{1}} d \rho_{2} r^{d-2}\left(\rho_{2}\right)\left[d-1-8 \pi G \phi^{2}\left(\rho_{2}\right)\right]
$$

Consider solutions with a large $r$ asymptotic such that $r(\rho) \rightarrow \infty$ and $r^{\prime}(\rho) \rightarrow$ const $>0$ as $\rho \rightarrow \infty$. Eq. (7) gives $r^{\prime}$ as $\int\left[r \phi^{\prime 2}\right] d \rho$, and its convergence as $\rho \rightarrow \infty$ implies a sufficiently rapid decay of $\phi^{\prime}$ at large $\rho$, so that $\phi \rightarrow \phi_{\infty}=$ const as $\rho \rightarrow \infty$. The potential $V$ then tends to a constant equal to $V\left(\phi_{\infty}\right)$. Furthermore, Eq. (8) shows that $A(\rho)$ can grow at large $r$ at most as $r^{2}$ and, lastly, substitution of the asymptotics of $\phi(\rho), A(\rho)$ and $r(\rho)$ into Eq. (6) leads to $d V / d \phi \rightarrow 0$ as $\rho \rightarrow \infty$. So the condition that there exists a large $r$ asymptotic, applied to the field equations, implies that at this asymptotic the scalar field tends either to an extremum of the potential $V(\phi)$, or to an inflection point with zero derivative. For the Mexican hat potential it can be either the maximum at $\phi=0$ (the trivial unstable solution for $\phi$ and de Sitter metric with the cosmological constant $2 \pi G \lambda \eta^{4}$ ) or a minimum of $V$, where $f=1$ and $V=0$. For a "slightly raised Mexican hat" (the potential (3) plus a small constant $V_{+}$) we have a de Sitter asymtotic with $f=1$ and $V=V_{+}$.

A regular center requires that $A=A_{c}+O\left(r^{2}\right)$ and $A r^{2} \rightarrow 1$ as $\rho \rightarrow \rho_{c}$ such that $r\left(\rho_{c}\right)=0$. Without loss of generality we put $\rho_{c}=0$ and $A_{c}=1$.

For the potential (3), regularity at $\rho=0$ and the asymptotic condition at $\rho \rightarrow \infty$ lead to $C_{1}=C_{2}=0$, and from (10) we have

$$
A(\rho)=2 r^{2}(\rho) \int_{\rho}^{\infty} \frac{d \rho_{1}}{r^{d+2}\left(\rho_{1}\right)} \int_{0}^{\rho_{1}} d \rho_{2} r^{d-2}\left(\rho_{2}\right)\left[d-1-8 \pi G \phi^{2}\left(\rho_{2}\right)\right] .
$$

Eq. (8) provides another representation for $A(\rho)$ satisfying the regular center conditions:

$$
A(\rho)=1-\frac{32 \pi G}{d} \int_{0}^{\rho} \frac{d \rho_{1}}{r^{d}\left(\rho_{1}\right)} \int_{0}^{\rho_{1}} d \rho_{2} r^{d}\left(\rho_{2}\right) V\left(\rho_{2}\right) .
$$

From (11) we find the limiting value of $A$ at $\rho \rightarrow \infty$ :

$$
A(\infty)=\frac{d-1-\gamma}{\alpha^{2}(d-1)}, \quad \gamma=8 \pi G \eta^{2},
$$

where $\alpha=d r / d \rho$ at $\rho \rightarrow \infty$ :

$$
\alpha=1-\frac{8 \pi G}{d} \int_{0}^{\infty} r(\rho) \phi^{\prime 2}(\rho) d \rho .
$$

Eq. (13) shows that $\gamma=d-1$ is a critical value of $\gamma$ : the large $r$ asymptotic can be static only if $\gamma \leq d-1$; for $\gamma<d-1$ it is flat up to a solid angle deficit, in full similarity to the conventional case $d=2$ [2] 1]. If $\gamma>d-1$, 
then $A(\infty)<0$, and there is a horizon at some $\rho=\rho_{h}$ where $A=0$. From (12),

$$
\frac{32 \pi G}{d} \int_{0}^{\rho_{h}} \frac{d \rho_{1}}{r^{d}\left(\rho_{1}\right)} \int_{0}^{\rho_{1}} d \rho_{2} r^{d}\left(\rho_{2}\right) V\left(\rho_{2}\right)=1,
$$

and so we have

$$
A(\rho)=-\frac{32 \pi G}{d} \int_{\rho_{h}}^{\rho} \frac{d \rho_{1}}{r^{d}\left(\rho_{1}\right)} \int_{0}^{\rho_{1}} d \rho_{2} r^{d}\left(\rho_{2}\right) V\left(\rho_{2}\right)
$$

The $\gamma$ dependence of $\rho_{h}$, where $\gamma=8 \pi G \eta^{2}$, can be found from the relation

$$
\frac{32 \pi G}{d} \int_{\rho_{h}}^{\infty} \frac{d \rho_{1}}{r^{d}\left(\rho_{1}\right)} \int_{0}^{\rho_{1}} d \rho_{2} r^{d}\left(\rho_{2}\right) V\left(\rho_{2}\right)=-\frac{d-1-\gamma}{\alpha^{2}(d-1)} .
$$

\section{2. $\quad$ Large $r$ asymptotic}

From (6) we can find the asymptotic behavior of the field $f(\rho)$ and the potential $V(\rho)$ at $r \rightarrow \infty$. Thus, at large $\rho$ we have $A \rightarrow A(\infty)$, see (13), and the field equation (6) reduces to

$$
\frac{1}{r^{d}} \frac{d}{d r}\left(r^{d} \frac{d f}{d r}\right)-\frac{d-1}{\gamma-d+1}\left[\lambda \eta^{2}\left(1-f^{2}\right)-\frac{d}{r^{2}}\right] f=0, \quad r \rightarrow \infty .
$$

A regular solution of this equation must tend to unity as $r \rightarrow \infty$, and for $\psi=1-f$ we have the linear equation

$$
\psi,_{r r}+\frac{d}{r} \psi,_{r}+\frac{2 \lambda \eta^{2}(d-1)}{\gamma-d+1}\left(\psi-\frac{d}{2 \lambda \eta^{2} r^{2}}\right)=0, \quad r \rightarrow \infty .
$$

The general solution of the corresponding homogeneous equation

$$
\psi_{0, r r}+\frac{d}{r} \psi_{0, r}+\frac{2 \lambda \eta^{2}(d-1)}{\gamma-d+1} \psi_{0}=0
$$

can be expressed in terms of Bessel functions:

$$
\psi_{0}(r)=r^{-(d-1) / 2}\left[C_{1} J_{-\frac{d-1}{2}}\left(\frac{r}{r_{0}}\right)+C_{2} Y_{-\frac{d-1}{2}}\left(\frac{r}{r_{0}}\right)\right], \quad r_{0}^{2}=\frac{\gamma-d+1}{2 \lambda \eta^{2}(d-1)} .
$$

A special solution of the inhomogeneous equation (16) at $r \rightarrow \infty$ is

$$
\psi=\frac{d}{2 \lambda \eta^{2} r^{2}}+O\left(\frac{1}{r^{4}}\right) .
$$

The general solution of Eq. (16) gives the following asymptotic behavior for the Higgs field magnitude $f$ as $r \rightarrow \infty$ :

$$
f(r)=1-\frac{d}{2 \lambda \eta^{2} r^{2}}-\frac{C}{\left(\lambda \eta^{2} r^{2}\right)^{d / 4}} \sin \left(\frac{r}{r_{0}}+\frac{\pi d}{4}+\varphi\right), \quad r \rightarrow \infty .
$$

Due to the boundary conditions imposed, the integration constants $C$ and $\varphi$ are functions of $d$ and $\gamma$ which can be found numerically. The function $C(\gamma)$ for $d=3$ is presented in Fig. 1. From (17) we find the asymptotic behavior of $V$ :

$$
V(r)=\frac{\lambda \eta^{4}}{4}\left[\frac{d}{\lambda \eta^{2} r^{2}}+\frac{2 C}{\left(\lambda \eta^{2} r^{2}\right)^{d / 4}} \sin \left(\frac{r}{r_{0}}+\frac{\pi d}{4}+\varphi\right)\right]^{2}, \quad r \rightarrow \infty .
$$

\subsection{Bounds of the classical regime and the monopole core}

Of certain interest are solutions with cosmological large $r$ behavior, i.e., those with $\gamma>d-1$. The latter condition means that the scalar field, approaching $\eta$ at large $r$, actually takes near- or trans-Planckian values.

Indeed, in $D$ dimensions, the Planck length $l_{D}$ and mass $m_{D}$ are expressed in terms of the gravitational constant $G=G_{D}$ as

$$
l_{D}=G_{D}^{1 / d}, \quad m_{D}=G_{D}^{-1 / d}, \quad d=D-2 .
$$

Therefore $\eta^{2}=\gamma /(8 \pi G)=\gamma m_{D}^{d} /(8 \pi)$, and, in the case of interest $\gamma \sim d$, we have

$$
\eta \sim\left(m_{D}\right)^{d / 2} \sqrt{d /(8 \pi)} .
$$




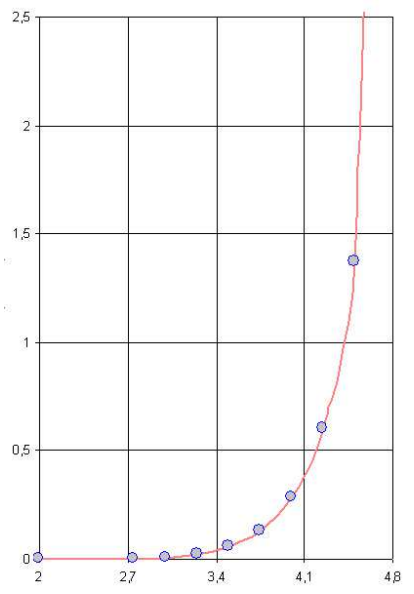

Figure 1: The function $C(\gamma)$ found numerically for $d=3$

We can, however, remain at sub-Planckian curvature values, thus avoiding the necessity to invoke quantum gravity, if we require sub-Planckian values of the potential $V$ in the whole space, i.e., $8 \pi G V=2 \pi G \lambda \eta^{4} \ll m_{D}^{2}$, whence it follows, for $\eta$ given by (19),

$$
\lambda \ll \frac{32 \pi}{d^{2}} m_{D}^{2-d} .
$$

We can thus preserve the classical regime even with large $\eta$ by choosing sufficiently small values of $\lambda$. In terms of lengths, this condition is equivalent to the requirement that the monopole core, radius $r_{\text {core }}=1 /(\sqrt{\lambda} \eta)$ is much greater than the Planck length:

$$
\frac{1}{\sqrt{\lambda} \eta} \gg l_{D}
$$

One may notice that this condition is external with respect to the theory since general relativity does not contain an internal restriction on the gravitational field strength. Moreover, in ordinary units, our dimensionless gravitational field strength parameter, expressed as $\gamma=8 \pi G c^{-4} \eta^{2}$, does not contain $\hbar$. Only when we compare the characteristic length existing in our theory, $r_{\text {core }}$, with the Planck length $l_{D}=\left(\hbar G / c^{3}\right)^{1 / d}$, we obtain the restriction (20) or (21).

Let us now discuss the solutions for $\gamma$ slightly exceeding the critical value $d-1$. In case $\gamma-(d-1) \ll 1$, the horizon radius $r_{h}$ is much greater than $r_{\text {core }}$, and the constant $C$ turns out to be negligibly small (this is confirmed numerically, see Fig. 1). Then the integrand in the internal integrals in (12), (14), (15) at large $\rho_{2}$ is

$$
d \rho_{2} r^{d}\left(\rho_{2}\right) V\left(\rho_{2}\right) \approx \frac{d^{2}}{4 \alpha \lambda} \frac{d r}{r^{4-d}} .
$$

The main contribution to the above internal integrals comes from the monopole core if $d<3$ and from the upper limit if $d>3$. In case $d=3$ it is a logarithmic integral. As a result, we have different behavior ${ }^{2}$ of $\rho_{h}(\gamma)$ at $\gamma-(d-1) \ll 1$ for $d=2$ and $d \geq 3$.

For $d=2$ (4-dimensional general relativity),

$$
\int_{0}^{\rho_{1}} d \rho_{2} r^{d}\left(\rho_{2}\right) V\left(\rho_{2}\right) \approx \int_{0}^{\infty} d \rho_{2} r^{2}\left(\rho_{2}\right) V\left(\rho_{2}\right)=\mathrm{const},
$$

and from (15) we find, in agreement with [1], that the horizon radius $r_{h}$ is inverse proportional to $\gamma-1$ :

$$
r_{h}=\mathrm{const} /(\gamma-1), \quad \gamma-1 \ll 1, \quad d=2 .
$$

For $d>3$ we find that at $\gamma-(d-1) \ll 1$ the horizon radius $r_{h}$ is inverse proportional to the square root of $\gamma-(d-1) \ll 1$ :

$$
r_{h}=\sqrt{\frac{\gamma d(d-1)}{2(d-3)(\gamma-d+1)} \frac{1}{\lambda \eta^{2}}}, \quad r_{h}^{2} \gg \frac{1}{\lambda \eta^{2}}, \quad d>3 .
$$

\footnotetext{
${ }^{2}$ This is the only important qualitative difference between the general case $d \geq 3$ and the particular case $d=2$, considered in 1 .
} 
It is thus confirmed that, for $\gamma-(d-1) \ll 1$, the horizon is located far from the monopole core, $r_{h}^{2} \gg 1 /\left(\lambda \eta^{2}\right)$. Then the function $A(r)$ at $r>r_{h}$ can be found analytically. In this case $r(\rho)$ is a linear function at $r>r_{h}$, and $d r=\alpha d \rho$. From (14) at $r>r_{h}$ we find

$$
A(r)=-\frac{\gamma+1-d}{\alpha^{2}(d-1)}\left(1-\frac{r_{h}^{d-1}}{r^{d-1}}\right)+\frac{\gamma d}{2 \alpha^{2}(d-3) \lambda \eta^{2} r^{2}}\left[1-\left(\frac{r_{h}}{r}\right)^{d-3}\right] .
$$

The condition of applicability of (23) is $l_{D} \ll r_{h}$. In view of $r_{\text {core }} \ll r_{h}$ it is less restrictive than the condition (21).

\subsection{Solutions with $f(\phi)$ changing sign}

As in Ref. 1, numerical integration of the field equations shows that, in addition to the solutions with totally positive (or totally negative) $f(u)$, there are also solutions with a regular center such that $f(u)$ changes its sign $n$ times where $n \geq 1$. All these solutions exist for $\gamma<\gamma_{n}(d)$, where $\gamma_{n}(d)$ are some critical values of the parameter $\gamma$. In case $n>0$, all of them have a horizon, and the absolute value of $f$ at the horizon $\left|f_{h, n}\left(\rho_{h}\right)\right|$ is a decreasing function of $\gamma$, vanishing as $\gamma \rightarrow \gamma_{n}-0$. Moreover, as $\gamma \rightarrow \gamma_{n}(d)$, the function $f(u)$ vanishes in the whole range $\rho \leq \rho_{h}$ and is small inside the horizon for $\gamma$ close to $\gamma_{n}(d)$. This allows us to find the critical values $\gamma_{n}(d)$ analytically: Eq. (6) reduces to a linear equation for $f$ in a given (de Sitter) background and, combined with the boundary conditions $f(0)=0$ and $f\left(\rho_{h}\right)<\infty$, leads to a linear eigenvalue problem. Its solution (see 1 for details) in the $d$-dimensional case gives the upper limits $\gamma_{n}(d)$ and the corresponding minimal horizon radii $r_{h}=r_{h n}$ for solutions with the Higgs field magnitude $f$ changing its sign $n$ times:

$$
\begin{aligned}
r_{h n} & =\sqrt{(2 n+1)(2 n+d+2) / \lambda \eta^{2}}, \\
\gamma_{n} & =\frac{2 d(d+1)}{(2 n+1)(2 n+d+2)} .
\end{aligned}
$$

For $d=2$ Eqs. (24), (25) reduce to (52) in Ref. 11. Under the condition (21) these solutions remain in the classical gravity regime.

\section{5-dimensional models and nonsingular cosmology}

\subsection{The extra dimension}

At present there is no evidence for the existence of more than three spatial dimensions up to the achievable energies about several hundred GeV. But this energy is quite tiny on the Planck scale, $\sim 10^{19} \mathrm{GeV}$. Our solutions of possible cosmological interest correspond to $\gamma>d-1$, i.e., the Planck energy scale. Even under the condition (21), there remains an enormous range of scales in the early Universe in which the number of equally important spatial dimensions could have been greater than 3 .

If we try to consider our $d=3$ solutions in a cosmological context, the extra coordinate is $t$ in (11) and (15). The coordinate $t$ is time inside the horizon and becomes a fourth spatial coordinate outside it, where $A(\rho)<0$. The metric (5) takes the form

$$
d s^{2}=\frac{d \rho^{2}}{|A(\rho)|}-|A(\rho)| d t^{2}-r^{2}(\rho) d \Omega_{3}^{2} .
$$

Introducing the proper time $\tau$ of a co-moving observer outside the horizon,

$$
\tau=\int_{\rho_{h}}^{\rho} \frac{d \rho}{\sqrt{|A(\rho)|}}
$$

we arrive at a 5-dimensional Kantowski-Sachs cosmology with a closed Friedman-Robertson-Walker metric in the $(3+1)$-dimensional space-time section of constant $t$ :

$$
d s_{4}^{2}=d \tau^{2}-a^{2}(\tau) d \Omega_{3}^{2}-|A(\rho(\tau))| d t^{2} .
$$

The 4-dimensional spherical radius $r(\rho)$ now plays the role of the scale factor: $a(\tau)=r(\rho(\tau))$.

It is tempting to explain the unobservability of the extra dimension parametrized by the coordinate $t$ by compactifying $t$ with a certain "period" $T$ in the spirit of Kaluza-Klein models. Such a compactification would, however, lead to a singularity at $r=r_{h}$, as is clear from Fig. 2 . If $t \in \mathbb{R}$, the static region (the left quadrant of the diagram) is connected with the future cosmological region (the upper quadrant) by the horizon, crossed by photons as well as massive particles without problems. If, however, the $t$ axis is made compact by identifying, say, the points $t_{1}$ and $t_{2}$ 


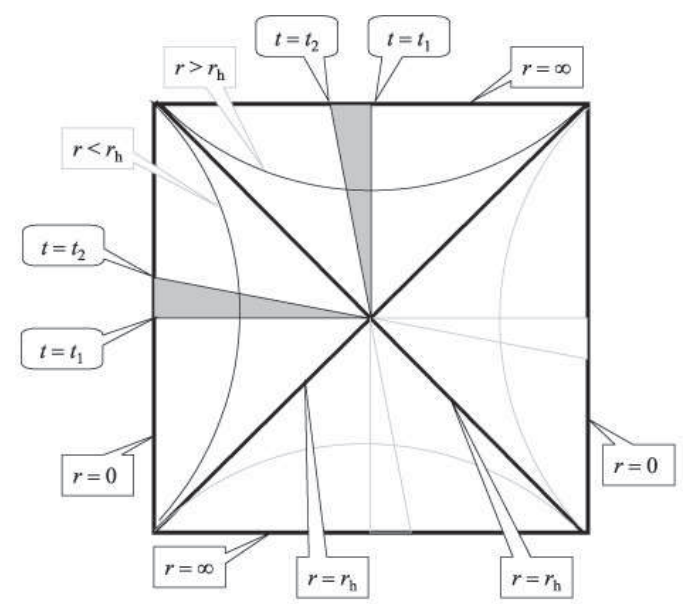

Figure 2: Carter-Penrose diagram of a global monopole with a cosmological horizon. The diagonals of the square depict the horizons. After identification of $t_{1}$ and $t_{2}$, only the dashed regions survive.

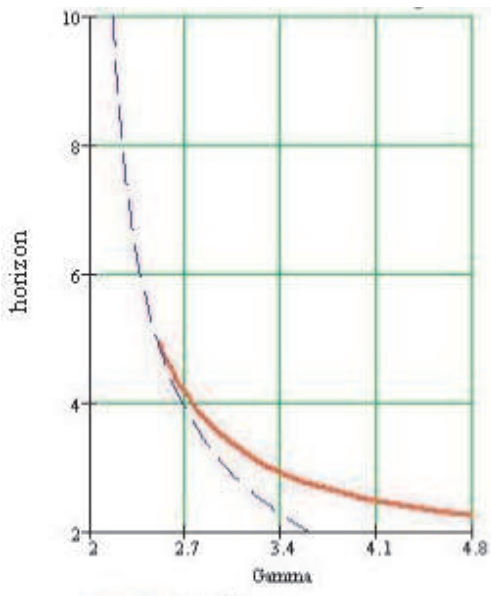

Figure 3: The dimensionless horizon radius $\sqrt{\lambda} \eta r_{h}$ vs. $\gamma$ for $d=3$ (solid line). The dashed line is the asymptotic dependence (28) valid for $\gamma-2 \ll 1$.

on the $t$ axis, then the static and cosmological regions in the diagram take the form of the dashed sectors, actually tubes of variable thickness, connected at one point only, the ends (tips) of the tubes. The curvature invariants do not change due to this identification and remain finite, and the emerging singularity in the $(\rho, t)$ plane resembles a conical singularity.

Compactification is not the only possibility of explaining why the $t$ coordinate is invisible. Instead one can assume that, at some instant of the proper cosmological time $\tau$ of the 5-dimensional model (27), a phase transition happens at a certain energy scale $1 / T$ leading to localization of matter on the 3 -spheres in the spirit of brane world models. Anyway, within our macroscopic theory without specifying the structure of the physical vacuum, it is impossible to explain why the extra dimension is not seen now. Nevertheless it is of interest to describe some cosmological characteristics of the $d=3$ global monopole.

\subsection{Some cosmological estimates}

For $d=3$ the internal integrals in (14) and (15) have a logarithmic character, and instead of (22) and (23) we get

$$
\gamma-2=\frac{3}{\lambda \eta^{2} r_{h}^{2}}\left[B+\ln \left(\lambda \eta^{2} r_{h}^{2}\right)\right], \quad r_{h}^{2} \gg \frac{1}{\lambda \eta^{2}}, \quad d=3
$$

and

$$
A(a)=-\frac{\gamma-2}{2 \alpha^{2}}\left(1-\frac{r_{h}^{2}}{a^{2}}\right)+\frac{3 \gamma}{2 \alpha^{2} \lambda \eta^{2}} \frac{\ln \left(a / r_{h}\right)}{a^{2}}, \quad a>r_{h}, \quad d=3
$$




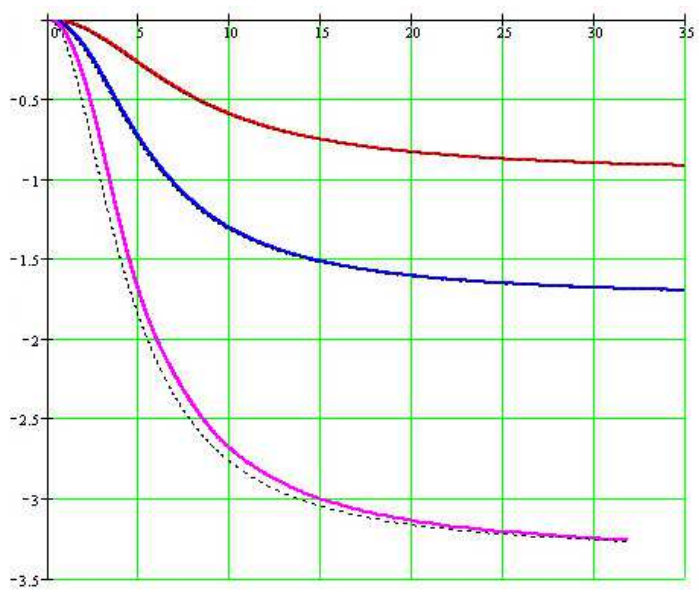

Figure 4: The function $A(\tau) \equiv A(a(\tau))$ for $d=3$ and $\gamma=3,3.5$ and 4. Solid lines show numerical results while dashed lines the analytical dependence (29).

The dependence $a(\tau)$ can be found from Eq.(26). In (28) $B$ is a constant about unity. Our numerical estimate gives $B \approx 0.75$. The dimensionless radius of the horizon $\sqrt{\lambda} \eta r_{h}$ as a function of $\gamma$ is presented in Fig. 3 for $d=3$ (solid line). The dashed line is the asymptotic dependence (28) valid for $\gamma-2 \ll 1$. The function $A(\tau) \equiv A(a(\tau))$ is shown in Fig. 4 for $d=3$ and $\gamma=3,3.5$ and 4 . The numerical and analytical results are shown by solid and dashed lines, respectively. It is remarkable that only for $\gamma=4$ the approximate analytical dependence (29), valid, strictly speaking, for $\gamma-2 \ll 1$, is slightly different from the more precise one found numerically.

Far outside the horizon $A(a)$ tends to a constant value:

$$
A(a) \rightarrow-\frac{\gamma-2}{2 \alpha^{2}}, \quad a \gg r_{h},
$$

and the metric (27) describes a uniformly expanding world with the linear dependence $a(\tau)$ at late times:

$$
a(\tau)=\alpha \sqrt{|A(\infty)|} \tau=\sqrt{\frac{\gamma-2}{2}} \tau, \quad \tau \rightarrow \infty .
$$

The Hubble parameter $H=\dot{a} / a$, where the dot denotes $d / d \tau$, is found analytically from the expression (29) for $A(a)\left[d=3, a>r_{h} \gg 1 /(\sqrt{\lambda} \eta)\right]:$

$$
H(a)=\frac{1}{a} \sqrt{\frac{\gamma-2}{2}\left(1-\frac{r_{h}^{2}}{a^{2}}\right)-\frac{3 \gamma}{2} \frac{\ln \left(a / r_{h}\right)}{\lambda \eta^{2} a^{2}} .}
$$

The temporal evolution of the Hubble parameter $H(\tau)$ is shown in Fig. 5 for $\gamma=3,3.5$, and 4 . The expansion starts from the horizon at $\tau=0$ and rather quickly approaches the late-time behavior $H(\tau)=\tau^{-1}$. We actually have the asymptotic regime almost immediately after the beginning.

If we try to extrapolate this late-time regime to the present epoch, we can use the estimate given in Ref. [1] (Box 27.4): $\dot{a} \approx 0.66$, and Eqs. (30) and (28) lead to

$$
\gamma=2+2 \dot{a}^{2}=2.87, \quad \sqrt{\lambda} \eta r_{h} \approx 3.65 .
$$

These estimates conform to the monopole parameter values leading to a nonsingular cosmology.

The symmetry-breaking potential (18), averaged over the oscillations, $V(\tau) \equiv \overline{V(a(\tau))}$ is a decreasing function of $\tau$ :

$$
V(\tau)=\frac{9}{(\gamma-2)^{2} \lambda \tau^{4}}+\frac{\lambda \eta^{4} C^{2}}{2\left[(\gamma / 2-1) \lambda \eta^{2} \tau^{2}\right]^{3 / 2}}, \quad \tau \rightarrow \infty .
$$

Scalar field potentials are often interpreted in cosmology as a time-dependent effective cosmological constant. A reason is that $V$ enters as a $\Lambda$-term into the energy-momentum tensor. As is seen from (33), in our case this term behaves as a mixture of two components, one decaying with cosmological expansion like radiation $\left(\sim \tau^{-4} \sim a^{-4}\right)$, the other like matter without pressure $\left(\sim \tau^{-3} \sim a^{-3}\right)$ in 4 dimensions. The four-dimensional energy density 


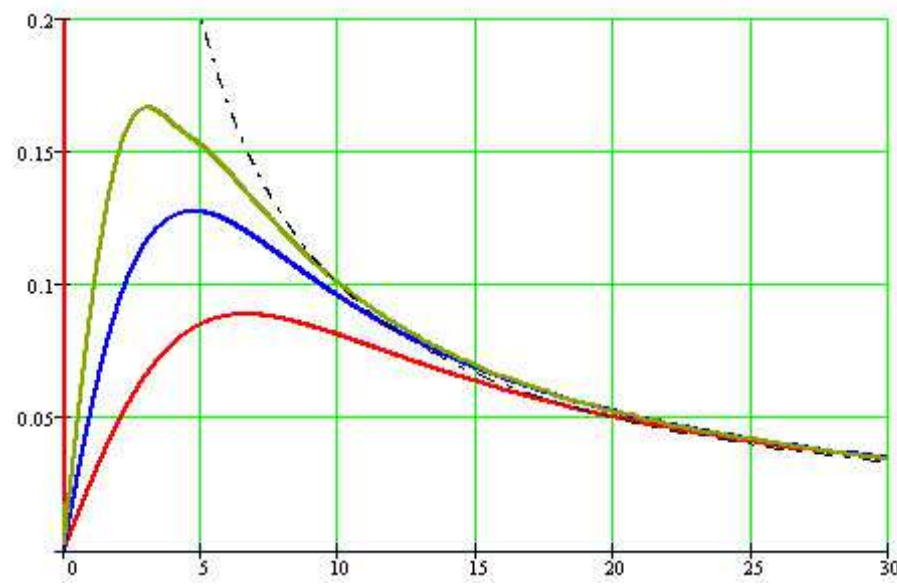

Figure 5: The Hubble parameter $H(\tau)$ for $\gamma=3,3.5$, and 4. At late times $H(\tau)=1 / \tau$ (dashed curve).

corresponding to $V$ is proportional to $V \sqrt{|A|}$. However, at late times $\sqrt{|A|}$, the extra-dimension scale factor, tends to a constant. Hence the five- and four-dimensional behaviours of the energy density actually coincide at large $\tau$. One can say that the potential $V(\phi)$ in the global monopole model gives rise to both dark radiation and dark matter. Recall that, by modern views, both must necessarily be present in the Universe from the observational viewpoint 12.

These estimates can only show that the 5-dimensional global monopole model is in principle able to give plausible cosmological parameters. Quantitative estimates certainly require a more complete model including further phase transitions, one of which should explain the unobservability of the fifth dimension.

\section{Acknowledgement}

The authors are grateful to A.F. Andreev, A.A. Starobinsky, V.A. Marchenko, and M. Yu. Kagan for useful discussions.

\section{References}

[1] K.A. Bronnikov, B.E. Meierovich and E.R. Podolyak, JETP 95, 392-403 (2002).

[2] A. Vilenkin and E.P.S. Shellard, "Cosmic Strings and Other Topological Defects" (Cambridge Univ. Press, Cambridge, 1994).

[3] S.L. Liebling, Phys. Rev. D 61, 024030 (1999).

[4] E.B.Gliner, Physics - Uspekhi 172, 221 (2002);

E.B. Gliner and I.G. Dymnikova, Physics - Uspekhi 172, 227 (2002).

[5] A. A. Starobinsky, Sov. Astron. Lett., 4, 82, (1978).

[6] V.A. Rubakov, "Large and infinite extra dimensions", Usp. Fiz. Nauk 171, 913 (2001) (in Russian); Phys. Usp. 44, 871 (2001); hep-ph/0104152

[7] R. Maartens, "Geometry and dynamics of the brane world", gr-qc/0101059

[8] D. Langlois, "Gravitation and cosmology in a brane universe", gr-qc/0207047

[9] I.G. Dymnikova, A. Dobosz, M.L. Fil'chenkov and A.A. Gromov, Phys. Lett., 506B, 351 (2001).

[10] K.A. Bronnikov and J.C. Fabris, JHEP 09, 062 (2002); hep-th/0207213

[11] C.W. Misner, K.S. Thorne and J.A. Wheeler, "Gravitation". (W. H. Freeman and Company, San Francisco, 1973).

[12] V. Sahni and A. Starobinsky, "The Case for a Positive Cosmological $\Lambda$-term", astro-ph/9904398

[13] K. Benson and I. Cho, "A universe in a global monopole", hep-th/0104067 